\title{
MIXTURES OF NONATOMIC MEASURES
}

\author{
K. P. S. BHASKARA RAO AND M. BHASKARA RAO
}

\begin{abstract}
In this paper, we answer the following question: Is a mixture of nonatomic measures nonatomic? The answer turns out to be no, in general. Four sufficient conditions are given under which mixtures become nonatomic.
\end{abstract}

1. Introduction and definitions. Let $(X, \mathscr{A})$ and $(Y, \mathscr{B})$ be two Borel structures. Let $P$ be a transition probability on $X \times \mathscr{B}$, i.e., $P$ is a function defined on $X \times \mathscr{B}$ taking values in $[0,1]$ such that $P(x, \cdot)$ is a probability measure on $\mathscr{B}$ for every $x$ in $X$ and $P(\cdot, B)$ is an $\mathscr{A}$-measurable function for every $B$ in $\mathscr{B}$. Let $\gamma$ be any probability measure on $\mathscr{A}$ and define $\mu(B)=\int P(x, B) \gamma(d x)$ for $B$ in $\mathscr{B} . \mu$ is a probability measure on $\mathscr{B}$, and is called the mixture of $P$ with respect to $\gamma . P$ is said to be nonatomic if $P(x, \cdot)$ is nonatomic for every $x$ in $X$.

$\$ 2$ gives an example of a nonatomic $P$ and a measure $\gamma$ such that the mixture of $P$ with respect to $\gamma$ is not nonatomic. $\$ 3$ gives four sufficient conditions under which all mixtures become nonatomic.

All measures considered in this paper are finite. A measure $\gamma$ on $\mathscr{A}$ is said to be two-valued if there is a real number $a$, positive, such that $\gamma(A)=0$ or $a$ for every $A$ in $\mathscr{A}$ and $\gamma(X)=a$. The terminology used here follows closely that of Neveu [2].

2. Example. Let $X$ be any uncountable set and $\mathscr{A}$, the countable cocountable $\sigma$-algebra on $X$. For each $x$ in $X$, let $\left(Y_{x}, \mathscr{B}_{x}, \mu_{x}\right)$ be a nonatomic probability space. Let $Y=\prod_{x \in X} Y_{x}$, the product space and $\mathscr{B}=\prod_{x \in X} \mathscr{B}_{x}$, the product $\sigma$-algebra. Fix $f_{0}$ in $Y$. Let $f_{0}^{x}=f_{0} / X-\{x\}$, the restriction of $f_{0}$ to $X-\{x\}$. For every $B$ in $\mathscr{B}$, define $B_{x}=f_{0}^{x}$-th section of $B=\left\{g(x) \in Y_{x}: g \in B\right.$ and $g=f_{0}$ on $\left.X-\{x\}\right\}$. It is easy to verify that $\left\{B_{x}: B \in \mathscr{B}\right\}=\mathscr{B}_{x}$. Now, $P: X \times \mathscr{B}$ into $[0,1]$ is defined as follows. $P(x, B)=$ $\mu_{x}\left(B_{x}\right)$. For every $x$ in $X, P(x, \cdot)$ is a nonatomic probability measure on $\mathscr{B}$. Since every $B$ in $\mathscr{B}$ is a countable-dimensional cylinder, it follows that $B_{x}=\varnothing$ for all but a countable number of $x$ 's or $=Y_{x}$ for all but a countable number of $x$ 's. Consequently, $P(x, B)=0$ for all but a

Received by the editors April 22, 1971 and, in revised form, June 23, 1971.

AMS 1969 subject classifications. Primary 2813, 2815; Secondary 6060.

Key words and fhrases. Atom of a $\sigma$-algebra, atom of a measure, nonatomic measure, transition probability, mixture, separable and Lindelöf topological spaces. 
countable number of $x$ 's or $=1$ for all but a countable number of $x$ 's. Hence, $P(\cdot, B)$ is $\mathscr{A}$-measurable for every $B$ in $\mathscr{B}$. Let $\gamma$ be the $0-1$ valued measure on $\mathscr{A}$ defined by $\gamma(A)=0$ or 1 according as $A$ is countable or cocountable. The mixture $\mu$ of $P$ with respect to $\gamma$ is $0-1$ valued and hence cannot be nonatomic. In fact, $\mu$ is a degenerate measure (at $f_{0}$ ).

3. THEOREM 1. If $\mathscr{B}$ is separable, i.e., has a countable generator, then the mixture $\mu$ of any nonatomic transition probability $P$ with respect to any probability measure $\gamma$ is nonatomic.

Proof. Since $\mathscr{B}$ is separable, any measure on $\mathscr{B}$ is nonatomic if and only if it is continuous. (A measure on $\mathscr{B}$ is said to be continuous if the measure of any atom of $\mathscr{B}$ is zero.) This can be proved by noting that there are no two-valued continuous measures on any separable $\sigma$-algebra. Since a mixture of continuous measures is continuous, our theorem follows.

THEOREM 2. If $\{P(x, \cdot): x$ in $X\}$ is a dominated family of nonatomic measures, i.e., there exists a $\sigma$-finite measure $\varphi$ on $\mathscr{B}$ such that $P(x, \cdot) \ll \varphi$ for every $x$ in $X$, then any mixture $\mu$ of $P$ is nonatomic.

Proof. By a theorem of Halmos and Savage (see, for example, [2, p. 112]), the family $\{P(x, \cdot): x$ in $X\}$ is equivalent to a countable subfamily $\left\{P\left(x_{n}, \cdot\right): n \geqq 1\right\}$, i.e., $P(x, B)=0$ for every $x$ in $X$ if and only if $P\left(x_{n}, B\right)=0$ for every $n \geqq 1$. If $\tau(\cdot)=\sum_{n \geqq 1}\left(1 / 2^{n}\right) P\left(x_{n}, \cdot\right)$, then the family $\{P(x, \cdot): x$ in $X\}$ is equivalent to $\tau$. By direct argument, it follows that $\tau$ is nonatomic. Further, the mixture $\mu$ is dominated by $\tau$. Hence, $\mu$ is nonatomic. (See, for example, [1, Theorem 2.4, p. 653].)

THEOREM 3. Let $X$ be a topological space having a countable dense set and $\mathscr{A}$ be the $\sigma$-algebra generated by open subsets of $X$. Further, assume that $P(\cdot, B)$ is a continuous function on $X$ for every $B$ in $\mathscr{B}$. If $P$ is nonatomic, then any mixture of $P$ is nonatomic.

In order to prove this theorem, we need the following lemmas.

Lemma 1. Let $\varphi$ be a nonatomic measure on $\mathscr{B}$ and $\tau$ be a two-valued measure on $\mathscr{B}$. Then $\varphi$ and $\tau$ are mutually singular.

Proof. By the Lebesgue decomposition theorem, we can write $\varphi=\varphi_{1}+\varphi_{2}$ where $\varphi_{1} \ll \tau$, and $\varphi_{2}$ and $\tau$ are mutually singular. Since $\tau$ is two-valued, $\varphi_{1}$ is also two-valued. As $\varphi_{1} \leqq \varphi, \varphi_{1}$ is also nonatomic. (See, for example, [1, p. 653].) Hence, $\varphi_{1}=0, \varphi_{2}=\varphi$ and so $\varphi$ and $\tau$ are mutually singular.

LEMMA 2. If $q_{1}, q_{2}, \cdots$ is a sequence of nonatomic measures and $\tau$ is a two-valued measure on $\mathscr{B}$, then there exists $B$ in $\mathscr{B}$ such that $\tau(B)=$ $\tau(Y)$ and $\varphi_{i}(B)=0$ for every $i \geqq 1$. 
Proof. Let $\varphi(\cdot)=\sum_{n \supseteq 1}\left(1 / 2^{n}\right)\left(1 / \varphi_{n}(Y)\right) \varphi_{n}(\cdot)$. Then, $\varphi$ is a nonatomic measure and hence by Lemma $1, \varphi$ and $\tau$ are mutually singular. So, there exists a $B$ in $\mathscr{B}$ such that $\tau(B)=\tau(Y)$ and $\varphi(B)=0$. This implies $\varphi_{i}(B)=0$ for every $i \geqq 1$.

Proof of Theorem 3. Let $\mu$ be a mixture of $P$ with respect to $\gamma$. Suppose $\mu$ is not nonatomic. Let $B_{0}$ be an atom of $\mu$. Since $\mu\left(B_{0}\right)>0$, the open set $U=\left\{x\right.$ in $\left.X: P\left(x, B_{0}\right)>0\right\}$ has positive $\gamma$-measure. As $X$ contains a dense denumerable set, $U$ contains a dense denumerable set, say, $x_{1}$, $x_{2}, \cdots$. The measure $\mu$ on $B_{0} \cap \mathscr{B}$ is two-valued. $P_{1}\left(x_{n}, \cdot\right)=P\left(x_{n}, \cdot\right) / B_{0}$; the restriction of $P\left(x_{n}, \cdot\right)$ to $B_{0} \cap \mathscr{B}$ is a sequence of nonatomic measures on $B_{0} \cap \mathscr{B}$. By Lemma 2 , there exists a $B$ in $\mathscr{B}, B \subset B_{0}$. such that $\mu(B)=$ $\mu\left(B_{0}\right)$ and $P\left(x_{n}, B\right)=0$ for every $n \geqq 1$. Let $C=\{x$ in $X: P(x, B)=0\}$. $C$ is a closed set and each $x_{n}$ is in $C$. So, $U \subset$ Closure of $\left\{x_{n}: n \geqq 1\right\} \subset C$. Now,

$$
\begin{aligned}
0 & \neq \mu\left(B_{0}\right)=\mu(B)=\int P(x, B) \gamma(d x) \\
& =\int_{U^{*}} P(x, B) \gamma(d x)+\int_{U^{c}} P(x, B) \gamma(d x)=0+0=0, \text { a contradiction. }
\end{aligned}
$$

That the second term is zero follows from the fact

$$
P(x, B) \leqq P\left(x, B_{0}\right)=0 \text { if } x \text { is in } U^{c} .
$$

THeOREM 4. Let $X$ be a Lindelöf topological space and $\mathscr{A}$ be the $\sigma$ algebra on $X$ generated by open subsets of $X$. Further, assume that $P(\cdot, B)$ is a continuous function on $X$ for every $B$ in $\mathscr{B}$. If $P$ is nonatomic, then any mixture of $P$ is nonatomic.

Proof. Let $\mu$ be a mixture of $P$ with respect to $\gamma$. Let $B_{0} \in \mathscr{B}$ be such that $\mu\left(B_{0}\right)$ is positive. Then $U=\left\{x\right.$ in $\left.X: P\left(x, B_{0}\right)>0\right\}$ is an open set of positive $\gamma$-measure. Write $U=\bigcup_{n \geqq 1} U_{n}$, where

$$
U_{n}=\left\{x \text { in } X: P\left(x, B_{0}\right) \geqq 1 / n\right\} \text {. }
$$

Since $\gamma(U)$ is positive, there exists $N$ such that $\gamma\left(U_{N}\right)$ is positive. Since $U_{N}$ is closed, it is Lindelöf. For every $B \subset B_{0}$, define

$$
U_{B}=\left\{x \text { in } X: P\left(x, B_{0}\right)>P(x, B)>1 / N+1\right\} .
$$

$U_{B}$ is an open set for every $B \subset B_{0}, B$ in $\mathscr{B}$. Now, $\left\{U_{B}: B \subset B_{0}\right.$ and $B$ in $\left.\mathscr{B}\right\}$ is an open cover of $U_{N}$. For, let $x$ be in $U_{N}$. Then $P\left(x, B_{0}\right) \geqq 1 / N>$ $1 / N+1$. By nonatomicity, there exists a $C$ in $\mathscr{B}, C \subset B_{0}$ such that $P\left(x, B_{0}\right)>$ $P(x, C)>1 / N+1$. Consequently, $x$ is in $U_{C}$. By the Lindelöf property of $U_{N}$, we can find a countable subcover for $U_{N}$ from $\left\{U_{B}: B \subset B_{0}\right.$ and $B$ in $\left.\mathscr{B}\right\}$. 
Hence, there exists $B \subset B_{0}, B$ in $\mathscr{B}$ such that $\gamma\left(U_{B}\right)$ is positive. On $U_{B}$, $1 / N+1<P(x, B)<P\left(x, B_{0}\right)$. So, $0<\mu(B)<\mu\left(B_{0}\right)$. This proves the nonatomicity of $\mu$.

REMARKS. (1) For the nonatomicity of $\mu$, the first theorem lays conditions on the Borel structure $(Y, \mathscr{B})$, the second on the family $\{P(x, \cdot): x$ in $X\}$ and the third and fourth on $(X, \mathscr{A})$ and on the family $\{P(\cdot, B): B$ in $\mathscr{B}\}$ of functions.

(2) For a related result see Theorem 1.2 of $[1$, p. 651].

(3) If one wishes to have a counterexample in the setup of a single Borel structure, one can construct one using the one given in $\S 2$.

The authors are thankful to Mr. D. Ramachandran for useful discussions regarding $\S 3$.

\section{REFERENCES}

1. R. A. Johnson, Atomic and nonatomic measures, Proc. Amer. Math. Soc. 25 (1970), 650-655.

2. J. Neveu, Bases mathématiques du calcul des probabilités, Masson, Paris, 1964; English transl., Holden-Day, San Francisco, Calif., 1965. MR 33 \#6659; MR 33 \#6660.

Indian Statistical Institute, Calcutta 35, India 